PROCEEDINGS OF THE

AMERICAN MATHEMATICAL SOCIETY

Volume 103, Number 1, May 1988

\title{
THE FENCHEL-MOREAU THEOREM FOR SET FUNCTIONS
}

\author{
HANG-CHIN LAI AND LAI-JUI LIN
}

(Communicated by Paul S. Muhly)

\begin{abstract}
The Fenchel-Moreau theorem for set functions is proved, and some properties of subdifferential and conjugate functional of set functions are investigated.
\end{abstract}

1. Introduction. Let $(X, \Gamma, \mu)$ be a finite atomless measure space, and $F$ a proper real-valued set function defined on $\Gamma$ (i.e. $F(\Omega)>-\infty$ for all $\Omega \in \Gamma$ and $F \not \equiv \infty)$. Let $\operatorname{Dom} F=\{\Omega \in \Gamma ; F(\Omega)$ is finite $\}$. The conjugate function $F^{*}$ of $F$ is defined by

$$
F^{*}(f)=\sup _{\Omega \in \Gamma}\left[\left\langle f, \chi_{\Omega}\right\rangle-F(\Omega)\right], \quad f \in L_{1}(X, \Gamma, \mu),
$$

and the biconjugate function $F^{* *}$ of $F$ is defined by

$$
F^{* *}(\Omega)= \begin{cases}\sup _{f \in L_{1}(X, \Gamma, \mu)}\left[\left\langle f, \chi_{\Omega}\right\rangle-F^{*}(f)\right] & \text { if } \Omega \in \operatorname{Dom} F, \\ +\infty & \text { if } \Omega \notin \operatorname{Dom} F .\end{cases}
$$

By the definitions of $F^{*}$ and $F^{* *}$, we easily get that

$$
F^{* *}(\Omega) \leq F(\Omega) \text { for all } \Omega \in \Gamma .
$$

The question arises that under what conditions, the equality in (3) holds. In [1] the classical Fenchel-Moreau theorem shows that a function $g$, defined on a topological vector space $U$, is convex and lower semicontinuous if and only if $g(x)=$ $g^{* *}(x)$ for all $x \in U$.

It is known that the Fenchel-Moreau theorem plays an important role in the theory of optimization; many authors investigate this theorem in more general cases, for example, one can consult Lai [6], Koshi and Komuro [7], Koshi, Lai, and Komuro [8], and Zowe [12]. All of these papers showed that the Fenchel-Moreau theorem holds for the functions defined on linear spaces. In this note, the function is considered on a $\sigma$-algebra $\Gamma$ of a measure space rather than on a linear space. There is a good deal of difference between the Fenchel-Moreau theorem for the set function on a $\sigma$-algebra and for the usual function on a linear space. In this note, some properties of subdifferential and conjugate functions of set functions are also established.

2. Preliminaries. Throughout this note, we assume that $(X, \Gamma, \mu)$ is a finite atomless measure space with $L_{1}(X, \Gamma, \mu)$ separable. Under these assumptions, for any $\Omega \in \Gamma$ with $\mu(\Omega)>0$, there exist a measurable set $\Lambda \subset \Omega$ with $\mu(\Lambda)>0$, and

Received by the editors September 29, 1986 and, in revised form, January 12, 1987.

1980 Mathematics Subject Classification (1985 Revision). Primary 26A51, 49A50, 90C25.

Key words and phrases. Convex set functions, conjugate and biconjugate set functions, weaklower (upper) semicontinuous set functions. 
a countable sequence $\left\{\Omega_{n}\right\}$ in $\Gamma$ such that $\left\{c_{n} \chi_{\Omega_{n}}\right\}$ is dense in $L_{1}(\Omega, \Gamma, \mu)$, where $c_{n} \in \mathbf{R}$, and $\chi_{\Omega_{n}}$ is the characteristic function of $\Omega_{n} \in \Gamma$.

DEFINITION 2.1. A set function $F: \Gamma \rightarrow \mathbf{R}$ is called convex if for any given $\lambda \in[0,1]$ and $\Omega, \Lambda \in \Gamma$, there exist sequences $\left\{\Omega_{n}\right\}$ and $\left\{\Lambda_{n}\right\}$ with $\chi_{\Omega_{n}} \stackrel{w^{*}}{\rightarrow} \lambda \chi_{\Omega \backslash \Lambda}$ and $\chi_{\Lambda_{n}} \stackrel{w^{*}}{\rightarrow}(1-\lambda) \chi_{\Lambda \backslash \Omega}$ such that

$$
\varlimsup_{n \rightarrow \infty} F\left(\Omega_{n} \cup \Lambda_{n} \cup(\Omega \cap \Lambda)\right) \leq \lambda F(\Omega)+(1-\lambda) F(\Lambda),
$$

where $\stackrel{w^{*}}{\rightarrow}$ stands for convergence in the weak ${ }^{*}$-topology. Since $(X, \Gamma, \mu)$ is a finite measure space, any set $\Omega \in \Gamma$ can be identified with a characteristic function $\chi_{\Omega}$ in $L_{1}(\chi, \Gamma, \mu)$. Hence one can regard $\Gamma$ as a subset $\chi_{\Gamma}=\left\{\chi_{\Omega}: \Omega \in \Gamma\right\}$ of $L_{1}(X, \Gamma, \mu)$. For each $f \in L_{1}(X, \Gamma, \mu)$, the integral $\int_{\Omega} f d \mu$ is identified with the dual pair $\langle f, \chi \Omega\rangle$.

DEFINITION 2.2[2]. A subfamily $\mathscr{S}$ of measurable subsets in $\Gamma$ is convex if for any $(\Omega, \Lambda, \lambda) \in \mathscr{S} \times \mathscr{S} \times[0,1]$, associated with sequences $\left\{\Omega_{n}\right\}$ and $\left\{\Lambda_{n}\right\}$ in $\Gamma$ with $\chi_{\Omega_{n}} \stackrel{w^{*}}{\rightarrow} \lambda \chi_{\Omega \backslash \Lambda}$ and $\chi_{\Lambda_{n}} \stackrel{w^{*}}{\rightarrow}(1-\lambda) \chi_{\Lambda \backslash \Omega}$, there exist subsequences $\left\{\Omega_{n_{k}}\right\}$ of $\left\{\Omega_{n}\right\}$ and $\left\{\Lambda_{n_{k}}\right\}$ of $\left\{\Lambda_{n}\right\}$ such that $\Omega_{n_{k}} \cup \Lambda_{n_{k}} \cup(\Omega \cap \Lambda) \in \mathscr{S}$ for all $k$.

DEFINITION 2.3. Let $\mathscr{S} \subset \Gamma$ be a convex subfamily of measurable sets. A set function $F: \mathscr{S} \rightarrow \mathbf{R}$ is called convex on $\mathscr{S}$ if for any given $\lambda \in[0,1]$ and $\Omega$, $\Lambda \in \mathscr{S}$, the following inequality holds:

$$
\varlimsup_{n \rightarrow \infty} F\left(\Omega_{n} \cup \Lambda_{n} \cup(\Omega \cap \Lambda)\right) \leq \lambda F(\Omega)+(1-\lambda) F(\Lambda)
$$

for any sequences $\left\{\Omega_{n}\right\},\left\{\Lambda_{n}\right\}$ with $\Omega_{n} \cup \Lambda_{n} \cup(\Omega \cap \Lambda) \in \mathscr{S}$ such that

$$
\chi_{\Omega_{n}} \stackrel{w^{*}}{\rightarrow} \lambda \chi_{\Omega \backslash \Lambda}, \quad \chi_{\Lambda_{n}} \stackrel{w^{*}}{\rightarrow}(1-\lambda) \chi_{\Lambda \backslash \Omega} .
$$

We define $w^{*}$-lower (-upper) semicontinuous and $w^{*}$-continuous of a set function at a point $\Omega$ in $\operatorname{Dom} F$ as follows:

DEFINITION 2.4. Let $F: \Gamma \rightarrow \mathbf{R} \cup\{+\infty\}$ be a set function with $\operatorname{Dom} F=\mathscr{S} \subset$ $\Gamma$.

(i) $F$ is called $w^{*}$-lower semicontinuous at $\Omega \in \mathscr{S}$ if

$$
-\infty<F(\Omega) \leq \lim _{n \rightarrow \infty} F\left(\Omega_{n}\right)
$$

for any sequence $\Omega_{n} \in \mathscr{S}$ with $\chi \Omega_{n} \stackrel{w^{*}}{\rightarrow} \chi \Omega$.

(ii) $F$ is called $w^{*}$-upper semicontinuous at $\Omega \in \mathscr{S}$ if

$$
\varlimsup_{n \rightarrow \infty} F\left(\Omega_{n}\right) \leq F(\Omega)<\infty
$$

for any sequence $\Omega_{n} \in \mathscr{S}$ with $\chi_{\Omega_{n}} \stackrel{w^{*}}{\rightarrow} \chi \Omega$.

(iii) $F$ is called $w^{*}$-continuous at $\Omega \in \mathscr{S}$ if

$$
F(\Omega)=\lim _{n \rightarrow \infty} F\left(\Omega_{n}\right)
$$

for any sequence $\left\{\Omega_{n}\right\}$ in $\mathscr{S}$ with $\chi \Omega_{n} \stackrel{w^{*}}{\rightarrow} \chi \Omega$.

DEFINITION $2.5[4]$. Let $\mathscr{S}$ be a convex subfamily of subsets in $\Gamma$ and $F: \mathscr{S} \rightarrow$ $\mathbf{R}$ a set function; a set $[F, \mathscr{S}]$ in $\mathbf{R} \times L_{1}(X, \Gamma, \mu)$, defined by

$$
\begin{array}{r}
{[F, \mathscr{S}]=w^{*} \text {-closure }\left\{\left(\gamma, \chi_{\Omega}\right) \in \mathbf{R} \times L_{\infty}(X, \Gamma, \mu) \mid \Omega \in \mathscr{S} \text { and } \varlimsup_{n \rightarrow \infty} F\left(\Omega_{n}\right) \leq \gamma\right.} \\
\text { for any sequence } \left.\Omega_{n} \in \mathscr{S} \text { with } \chi_{\Omega_{n}} \stackrel{w^{*}}{\rightarrow} \chi_{\Omega}\right\},
\end{array}
$$

is called the epigraph of $F$ on $\mathscr{S}$. 
DEFINITION 2.6 [5]. An element $f \in L_{1}(X, \Gamma, \mu)$ is called a subgradient of a convex set function $G$ at $\Omega_{0} \in \Gamma$ if it satisfies the inequaltiy

$$
G(\Omega) \geq G\left(\Omega_{0}\right)+\left\langle f, \chi_{\Omega}-\chi_{\Omega_{0}}\right\rangle \text { for all } \Omega \in \Gamma .
$$

Note that the subgradient of a convex set function at a point $\Omega_{0}$ is not unique; usually it is a set of the following form:

$$
\partial G\left(\Omega_{0}\right)=\left\{f \in L_{1}(X, \Gamma, \mu): G(\Omega) \geq G\left(\Omega_{0}\right)+\left\langle f, \chi_{\Omega}-\chi_{\Omega_{0}}\right\rangle \text { for all } \Omega \in \Gamma\right\} .
$$

The set $\partial G\left(\Omega_{0}\right)$ is called the subdifferential of $G$ at $\Omega_{0}$, and if $\partial G\left(\Omega_{0}\right) \neq \varnothing, G$ is said to be subdifferential at $\Omega_{0}$. The subdifferential of a conjugate functional $G^{*}$ at $f_{0} \in L_{1}(X, \Gamma, \mu)$ is defined by a subfamily of measurable subsets in $\Gamma$ as follows:

$$
\partial G^{*}\left(f_{0}\right)=\left\{\Omega \in \Gamma: G^{*}(f) \geq G^{*}\left(f_{0}\right)+\left\langle f-f_{0}, \chi_{\Omega}\right\rangle \text { for all } f \in L_{1}(X, \Gamma, \mu)\right\} .
$$

It is remarkable that $\partial G^{*}$ is some different from [5].

\section{Main results.}

LEMMA $3.1[4,(3)]$. If, for any given sets $\Omega, \Lambda \in \Gamma, \lambda \in[0,1]$, and $L_{\infty}(X, \Gamma, \mu)$-sequences $\left\{\chi_{\Omega_{n}}\right\}$ and $\left\{\chi_{\Lambda_{n}}\right\}$,

$$
\chi_{\Omega_{n}} \stackrel{w^{*}}{\rightarrow} \lambda \chi_{\Omega \backslash \Lambda}, \quad \chi_{\Lambda_{n}} \stackrel{w^{*}}{\rightarrow}(1-\lambda) \chi_{\Lambda \backslash \Omega}
$$

then

$$
\chi_{\Omega_{n} \cup \Lambda_{n} \cup(\Omega \cap \Lambda)} \stackrel{w^{*}}{\rightarrow} \lambda \chi_{\Omega}+(1-\lambda) \chi_{\Lambda} .
$$

A subset $A \subset \mathbf{R} \times \chi_{\Gamma}$ is said to be convex if, for $\left(r, \chi_{\Omega}\right),\left(s, \chi_{\Lambda}\right) \in A$ and $\lambda \in[0,1]$, there exist sequences $V_{n}(\lambda)=\Omega_{n} \cup \Lambda_{n} \cup(\Omega \cap \Lambda)$ in $\Gamma$ and $t_{n}$ in $\mathbf{R}$ such that (4) holds and $t_{n} \rightarrow \lambda r+(1-\lambda) s$.

LEMMA $3.2[\mathbf{4}$, PROPOSITION 1]. Let $\mathscr{S}$ be a convex subset of $\Gamma$ and $F: \mathscr{S}$ $\rightarrow \mathbf{R}$ a convex set function. Then $[F, \mathscr{S}]$ is convex.

We modify the proof of this lemma given in [4] as follows:

PROOF. Let

$$
A=\left\{\left(r, \chi_{\Omega}\right) \mid \Omega \in \mathscr{S} \text { and } \varlimsup F\left(\Omega_{n}\right) \leq r\right\},
$$

where $\left\{\Omega_{n}\right\}$ is any sequence in $\mathscr{S}$ such that $\chi_{\Omega_{n}} \stackrel{w^{*}}{\rightarrow} \chi_{\Omega}$. Then $A$ is convex. In fact, for $\left(r, \chi_{\Omega}\right),\left(s, \chi_{\Lambda}\right)$ in $A$ and $\lambda \in[0,1]$, since $F$ is convex on the convex subfamily $\mathscr{S}$, there exists a sequence $V_{n}(\lambda)=\Omega_{n} \cup \Lambda_{n} \cup(\Omega \cap \Lambda)$ in $\mathscr{S}$ associated with $(\Omega, \Lambda, \lambda) \in \mathscr{S} \times \mathscr{S} \times[0,1]$ such that

$$
\varlimsup_{n \rightarrow \infty} F\left(V_{n}\right) \leq \lambda F(\Omega)+(1-\lambda) F(\Lambda) \leq \lambda r+(1-\lambda) s .
$$

Thus there is a subsequence $\left\{V_{n_{i}}\right\}$ of $\left\{V_{n}\right\}$ such that

$$
F\left(V_{n_{i}}(\lambda)\right) \leq \lambda r+(1-\lambda) s+1 / i \equiv t_{i}
$$

say. Hence for any sequence $\left\{U_{k}\right\}$ in $\mathscr{S}$ with $U_{k} \stackrel{w^{*}}{\rightarrow} V_{n_{i}}$, and from the $w^{*}$-upper semicontinuity of $F$ when $F$ is convex (see Lemma 3.3), we have $\varlimsup_{k \rightarrow \infty} F\left(U_{k}\right) \leq$ $F\left(V_{n_{i}}\right) \leq t_{i}$. So $\left(t_{i}, V_{n_{i}}\right) \in A$ and $\chi_{V_{n_{i}}} \rightarrow \lambda \chi_{\Omega}+(1-\lambda) \chi_{\Lambda}, t_{i} \rightarrow \lambda r+(1-\lambda) s$ (as $i \rightarrow \infty)$. This shows that $A$ is convex. It follows that the $w^{*}$-closure convex hull of $A$ coincides with the $w^{*}$-closure $\bar{A}=[\mathscr{S}, F]$ of $A$ in $\mathbf{R} \times L^{\infty}$. Hence $[\mathscr{S}, F]$ is convex. Q.E.D. 
LEMMA 3.3. Let $\mathscr{S} \subset \Gamma$ be a convex subfamily in $\Gamma$. Then any convex set function $F: \mathscr{S} \rightarrow \mathbf{R}$ is $w^{*}$-upper semicontinuous on $\mathscr{S}$.

ProOF. For any $\Omega \in \mathscr{S}$, let $\left\{\Omega_{n}\right\}$ be a sequence in $\mathscr{S}$ such that

$$
\chi_{\Omega_{n}} \stackrel{w^{*}}{\rightarrow} \chi_{\Omega}=\chi_{\Omega \backslash \varnothing}
$$

where $\varnothing$ is the empty set. Let each $\Lambda_{n}=\varnothing$. Then $\chi_{\Lambda_{n}} \stackrel{w^{*}}{\rightarrow}(1-1) \chi \varnothing \backslash \Omega$. It follows from Lemma 3.1 that

$$
\begin{aligned}
\varlimsup_{n \rightarrow \infty} F\left(\Omega_{n}\right) & =\varlimsup_{n \rightarrow \infty} F\left(\Omega_{n} \cup \Lambda_{n} \cup(\Omega \cap \varnothing)\right) \\
& \leq F(\Omega)+(1-1) F(\varnothing)=F(\Omega) .
\end{aligned}
$$

This shows that $F$ is $w^{*}$-upper semicontinuous on $\mathscr{S}$. Q.E.D.

From Lemma 3.3, the following corollary is immediate.

COROLlaRY 3.4. Let $\mathscr{S} \subseteq \Gamma$ be a convex subfamily of subsets. Then any $w^{*}$-lower semicontinuous set function $F: \mathscr{S} \rightarrow \mathbf{R}$ is $w^{*}$-continuous.

THEOREM 3.5. Let $F: \Gamma \rightarrow \mathbf{R} \cup\{\infty\}$ be a proper convex set function on $\Gamma, w^{*}$ lower semicontinuous on its convex domain $\mathscr{S}$. Then $\partial F(\Omega) \neq \varnothing$ and $\operatorname{Dom} F^{*} \neq$. $\varnothing$.

ProOF. It follows from Corollary 3.4 that $F$ is $w^{*}$-continuous on $\mathscr{S}$. Thus for any $\Lambda \in \mathscr{S}$, there is a sequence $\Lambda_{n}$ in $\mathscr{S}$ with $\chi_{\Lambda_{n}} \stackrel{w^{*}}{\rightarrow} \chi_{\Lambda}$ such that $\varlimsup_{n \rightarrow \infty} F\left(\Lambda_{n}\right)$ $=\lim _{n \rightarrow \infty} F\left(\Lambda_{n}\right)=F(\Lambda)$ and $\left(F(\Lambda), \chi_{\Lambda}\right) \in[F, \mathscr{S}]$ for all $\Lambda \in \mathscr{S}$. In view of Lemma 3.2 and the definition of epigraph, it has been proved that the epigraph $[F, \mathscr{S}]$ of $F$ is convex and $w^{*}$-closed. Since for any $r<F(\Omega),\left(r, \chi_{\Omega}\right) \notin[F, \mathscr{S}]$, and from the separation theorem, it follows that there exists a nonzero continuous linear functional $(-\alpha, f) \in \mathbf{R} \times L_{1}(X, \Gamma, \mu)$ such that

$$
\left\langle f, \chi_{\Lambda}\right\rangle-\alpha C<\left\langle f, \chi_{\Omega}\right\rangle-\alpha r \text { for all }\left(C, \chi_{\Lambda}\right) \in[F, \mathscr{S}] \text {. }
$$

In particular, when $\Lambda=\Omega$ and $C=F(\Omega)$, it is deduced that $\alpha(F(\Omega)-r)>0$. Since $r<F(\Omega)$ is arbitrary, $\alpha>0$. Hence

$$
\left\langle f, \chi_{\Lambda}\right\rangle-\alpha C \leq\left\langle f, \chi_{\Omega}\right\rangle-\alpha F(\Omega) \text { for all }\left(C, \chi_{\Lambda}\right) \in[F, \mathscr{S}] .
$$

Take $C=F(\Lambda)$. When both sides of the inequality (5) are divided by $\alpha>0$, we then obtain

$$
\left\langle f / \alpha, \chi_{\Lambda}\right\rangle-F(\Lambda) \leq\left\langle f / \alpha, \chi_{\Omega}\right\rangle-F(\Omega) \text { for all } \Lambda \in \mathscr{S} \text {. }
$$

As $F$ is a proper set function, inequality (6) holds for all $\Omega \in \Gamma$. By taking the supremum over all $\Lambda \in \Gamma$, we get

$$
F^{*}(f / \alpha) \leq\left\langle f / \alpha, \chi_{\Omega}\right\rangle-F(\Omega)
$$

Since (from (6))

$$
F(\Lambda) \geq F(\Omega)+\left\langle f / \alpha, \chi_{\Lambda}-\chi_{\Omega}\right\rangle \text { for all } \Lambda \in \Gamma,
$$

it follows that $f / \alpha \in \partial F(\Omega) \neq \varnothing$ and $f / \alpha \in \operatorname{Dom} F^{*} \neq \varnothing$. Q.E.D.

The following theorem is the Fenchel-Moreau theorem for set functions. 
THEOREM 3.6. Let $F$ be a proper convex $w^{*}$-lower semicontinuous set function on its convex domain $\mathscr{S}$. Then $F(\Omega)=F^{* *}(\Omega)$ for all $\Omega \in \Gamma$.

PROOF. It follows from Corollary 3.4 that $F$ is $w^{*}$-continuous on $\mathscr{S}$. A similar argument with the proof of Theorem 3.5 , we see that $\left(F(\Omega), \chi_{\Omega}\right) \in[F, \mathscr{S}]$ for all $\Omega \in \mathscr{S}$.

For any $r<F(\Omega)$, then the pair $\left(r, \chi_{\Omega}\right) \notin[F, \mathscr{S}]$. Since $\mathscr{S}$ is a convex subfamily of $\Gamma$ and $F: \mathscr{S} \rightarrow \mathbf{R}$ is a convex set function, $[F, \mathscr{S}]$ is a convex $w^{*}$-closed subset of $\mathbf{R} \times L_{\infty}(X, \Gamma, \mu)$. Applying the separation theorem, we can find a nonzero functional $(\alpha, f) \in \mathbf{R} \times L_{1}(X, \Gamma, \mu)$ which strictly separates the point $\left(r, \chi_{\Omega}\right)$ and the epigraph $[F, \mathscr{S}]$. Thus there exists $\varepsilon>0$ such that

$$
\sup _{\left(\lambda, \chi_{\Omega}\right) \in[F, \mathcal{P}]}\left\langle(\alpha, f),\left(\lambda, \chi_{\Lambda}\right)\right\rangle \leq\left\langle(\alpha, f),\left(r, \chi_{\Omega}\right)\right\rangle-\varepsilon
$$

It follows that

$$
\left\langle f, \chi_{\Lambda}\right\rangle+\lambda \alpha \leq\left\langle f, \chi_{\Omega}\right\rangle+\alpha r-\varepsilon
$$

for $\Lambda \in \mathscr{S}, \lambda \geq F(\Lambda)$. Note that $\alpha \leq 0$; for otherwise letting $\lambda \rightarrow \infty$, it would reduce to a contradiction. Actually, $\alpha<0$. For if $\Omega \in \operatorname{Dom} F$, then letting $\Lambda=\Omega$ and $\lambda=F(\Omega)$, we reduce from $(7)$ that $-\alpha(F(\Omega)-r) \geq \varepsilon>0$. Since $r<F(\Omega)$, it follows that $\alpha<0$. Next, let $\lambda=F(\Lambda)$ and then divide both sides of (7) by $-\alpha$. We obtain

$$
\left\langle\bar{f}, \chi_{\Lambda}\right\rangle-F(\Lambda) \leq\left\langle\bar{f}, \chi_{\Omega}\right\rangle-r+\varepsilon / \alpha
$$

where $\bar{f}=f /-\alpha$. By taking the supremum over $\Lambda \in \Gamma$, we obtain

$$
F^{*}(\bar{f}) \leq\left\langle\bar{f}, \chi_{\Omega}\right\rangle-r+\varepsilon / \alpha<\infty
$$

and so $\bar{f} \in \operatorname{Dom} F^{*}$. Thus

$$
r<r-\varepsilon / \alpha \leq\left\langle\bar{f}, \chi_{\Omega}\right\rangle-F^{*}(\bar{f}) \leq F^{* *}(\Omega) .
$$

This shows that for any $r<F(\Omega)$, we have

$$
r<F^{* *}(\Omega) \text {. }
$$

As we take $r=F(\Omega)-\delta$ for any given $\delta>0$, we have

$$
r=F(\Omega)-\delta<F(\Omega)
$$

It follows from inequality (8) that

$$
F(\Omega)-\delta<F^{* *}(\Omega) \text { or } F(\Omega)<F^{* *}(\Omega)+\delta .
$$

Since $\delta$ is arbitrary,

$$
F(\Omega) \leq F^{* *}(\Omega) \text { for all } \Omega \in \mathscr{S} .
$$

Consequently, from (9) and (3), we obtain

$$
F^{* *}(\Omega)=F(\Omega) \text { for all } \Omega \in \operatorname{Dom} F=\mathscr{S} \text {. }
$$

While $\Omega \notin \operatorname{Dom} F=\mathscr{S}, F(\Omega)=F^{* *}(\Omega)=\infty$. Hence

$$
F(\Omega)=F^{* *}(\Omega) \text { for all } \Omega \in \Gamma \text {. Q.E.D. }
$$

By the same argument used in [5], the following lemma is immediate. 
LEMMA 3.7. Let $F$ be a convex set function and $F^{*}$ the conjugate function of $F$. Let $f_{0} \in \operatorname{Dom} F^{*}$ and $\Omega_{0} \in \operatorname{Dom} F$. Then

$$
f_{0} \in \partial F\left(\Omega_{0}\right) \text { if and only if } F\left(\Omega_{0}\right)+F^{*}\left(f_{0}\right)=\left\langle f_{0}, \chi_{\Omega_{0}}\right\rangle .
$$

Using Theorem 3.6, we obtain

THEOREM 3.8. Let $F$ be proper convex $w^{*}$-lower semicontinuous set function on its convex domain $\mathscr{S}$. If $f_{0} \in \operatorname{Dom} F^{*}$ and $\Omega_{0} \in \mathscr{S}$, then

$$
f_{0} \in \partial F\left(\Omega_{0}\right) \text { if and only if } \Omega_{0} \in \partial F^{*}\left(f_{0}\right) \text {. }
$$

ProOF. It follows from Lemma 3.7 and Theorem 3.6 that

$$
f_{0} \in \partial F\left(\Omega_{0}\right) \text { if and only if } F\left(\Omega_{0}\right)+F^{*}\left(f_{0}\right)=\left\langle f_{0}, \chi_{\Omega_{0}}\right\rangle \text {, }
$$

that is, $F^{* *}\left(\Omega_{0}\right)+F^{*}\left(f_{0}\right)=\left\langle f_{0}, \chi_{\Omega_{0}}\right\rangle$ and $\Omega_{0} \in \partial F^{*}\left(f_{0}\right)$. Q.E.D.

\section{REFERENCES}

1. J. P. Aubin, Mathematical method of game and economic theory, North-Holland, Amsterdam, 1979.

2. J. H. Chou, W. S. Hsia, and T. Y. Lee, On multiple objective programming problems with set functions, J. Math. Anal. Appl. 105 (1985), 383-394.

3. _ Second order optimality conditions for mathematical programming with set functions, J. Austral. Math. Soc. (Ser. B) 26 (1985), 284-292.

4. H. C. Lai, S. S. Yang, and George R. Hwang, Duality in mathematical programming of set functions "On Fenchel duality theorem", J. Math. Anal. Appl. 95 (1983), 223-234.

5. H. C. Lai and S. S. Yang, Saddle point and duality in the optimization theory of convex set functions, J. Austral. Math. Soc. (Ser. B) 24 (1982), 130-137.

6. H. C. Lai, Conjugate operators and subdifferentials for convex operators in ordered vector space, Soochow J. Math. 9 (1983), 127-135.

7. S. Koshi and N. Komuro, A generalization of the Fenchel-Moreau theorem, Proc. Japan Acad. (Ser. A) 59 (1983), 178-181.

8. S. Koshi, H. C. Lai, and N. Komuro, Convex programming on spaces of measurable functions, Hokkaido Math. J. 14 (1985), 75-84.

9. D. G. Luenberger, Optimization by vector space methods, Wiley, New York, 1969.

10. R. J. T. Morris, Optimization problems involving set functions, Ph.D. dissertation, Univ. of California, Los Angeles, March 1978; also available as UCLA Tech. Report UCLA-ENG7815, March 1978.

11. __ Optimal constrained selection of measurable subset, J. Math. Anal. Appl. 70 (1979), 546-562.

12. J. Zowe, A duality theorem for a convex programming in ordered complete vector lattics, J. Math. Anal. Appl. 50 (1975), 274-287.

Department of Mathematics, University of IOWA, IOWA City, IOWA 52242

INSTitute of Mathematics, National TSING HUA UNIVERSity, Hoinchu, TAIWAN, REPUBLIC OF CHINA

Department of Mathematics, National Taiwan College of Education, Changhua, Taiwan, Republic of China 